\title{
ABSORBING BOUNDARY CONDITIONS FOR THE MULTIDIMENSIONAL KLEIN-GORDON EQUATION*
}

\author{
HOUDE HAN ${ }^{\dagger}$ AND DONGSHENG YIN $\ddagger$
}

\begin{abstract}
We consider the numerical solution of the linear Klein-Gordon equation in $\mathbb{R}^{2}$ and $\mathbb{R}^{3}$. An artificial boundary is introduced to obtain a bounded computational domain. On the given artificial boundary, the exact boundary condition and a series of approximating boundary conditions are constructed, which are called absorbing boundary conditions. By using either the exact or approximating boundary conditions on the artificial boundary, the original problem is reduced to either an equivalent or an approximately equivalent initial-boundary value problem on the bounded computational domain. The uniqueness of the approximate problem is then proved. The numerical results demonstrate that the method given in this paper is effective and feasible.
\end{abstract}

Key words. Klein-Gordon equation, artificial boundary, absorbing boundary condition

AMS subject classifications. 35L05 35Q40 42C10 65M60

\section{Introduction}

We consider the absorbing boundary conditions for the Klein-Gordon equation in $\mathbb{R}^{d}(d=2,3)$. The Klein-Gordon equation is given by

$$
\hbar^{2} \frac{\partial^{2} u}{\partial t^{2}}-\hbar^{2} c^{2} \Delta u+m^{4} c^{4} u=f(x, t),
$$

where $\hbar$ is the Planck constant, $m$ is the mass of the particle, and $c$ is the velocity of the particle.

The Klein-Gordon equation is a basic equation in relativistic quantum mechanics [10] which describes the behavior of spin-zero particles, like pions or kaons.

For simplicity, we consider the following initial value problem:

$$
\begin{aligned}
& \frac{\partial^{2} u}{\partial t^{2}}-\Delta u+u=f(x, t), \quad \forall(x, t) \in \mathbb{R}^{d} \times(0, T], \\
& \left.u\right|_{t=0}=\varphi_{0}(x),\left.\quad u_{t}\right|_{t=0}=\varphi_{1}(x), \forall x \in \mathbb{R}^{d} .
\end{aligned}
$$

We assume that $\varphi_{0}(x), \varphi_{1}(x)$, and $f(x, t)$ satisfy

$$
\begin{aligned}
& \operatorname{supp}\left\{\varphi_{0}(x)\right\} \subset B_{R_{0}}=\left\{x|| x \mid \leq R_{0}\right\}, \\
& \operatorname{supp}\left\{\varphi_{1}(x)\right\} \subset B_{R_{0}}, \\
& \operatorname{supp}\{f(x, t)\} \subset B_{R_{0}} \times[0, T] .
\end{aligned}
$$

Because the physical domain is unbounded, a numerical scheme for solving (1.1)-(1.2) requires us to bound the computational domain by imposing an artificial boundary condition on a given artificial boundary. Jin.

*Received: May 21, 2007; accepted (in revised version): July 25, 2007. Communicated by Shi

$\dagger$ Department of Mathematical Sciences, Tsinghua University, Beijing 100084, P.R. China (dyin@math.tsinghua.edu.cn).

${ }^{\ddagger}$ Department of Mathematical Sciences, Tsinghua University, Beijing 100084, P.R. China (hhan@math.tsinghua.edu.cn).

This work is supported in part by NSFC for Project 10471073 and China Postdoctoral Science Foundation 20060390076. 
Artificial boundary condition methods (ABC methods) refer to a class of methods that is based on specifying proper boundary conditions on the artificial boundary. The terms "radiation", "non-reflecting", or "absorbing" boundary conditions are often used in the context of exterior wave problems.

There are two different approaces to constructing artificial boundary conditions for wave equations. The first approach is called the perfectly matching layers method $[4,18]$; the methods in this class are often very effective. However, there are few theoretical results about their accuracy, convergence rate and computational costs. The second approach is called the transparent or absorbing boundary conditions method $[5,7,11,12,14]$, for which the resulting boundary-value problem is either approximates or is equivalent to the original problem. Some classic examples of these approaches are mentioned below.

In a well-known paper [5], Engquist and Majda explained local transparent boundary conditions and furthermore extended the idea to equations with variable coefficients and to domains with circular artificial boundaries. Bayliss and Turkel [3] obtained a series of artificial boundary conditions based on a asymptotic expansion of solutions to the hyperbolic equation at large distances. Higdon [17] considered a two-dimensional wave equation in a rectangular computational domain.

Fully local boundary conditions are used in practice, however, they may generate nonphysical reflections at an artificial boundary. Moreover, the well-posedness of the resulting truncated initial-boundary problem is unknown. While the using discrete transparent boundary conditions is very efficient, it has some disadvantages. Most of the discrete transparent boundary conditions are of second-order accuracy, and the computational meshes must be uniform either in time, in space, or both. Moreover, the discrete boundary conditions are still nonlocal. Many methods have been introduced to construct high-order local nonreflecting boundary conditions, see [8] and references therein.

Nonlocal artificial boundary conditions have the potential of being more accurate than local ones. Ting and Miksis [22] used the Kirchhoff formula for reflected waves from the scatter to propose an exact boundary condition for scattering problems. Teng [21] derived an exact nonreflecting boundary condition based on a boundary integral equation for exterior problems of the three dimensional wave equation. Teng's method has advantages as it only involves information from a finite amount of past time and has the flexibility of artificial boundary controls. Sofronov [20] and Grote and Keller [11] designed an exact boundary condition on a spherical boundary for the three dimensional wave equation by decomposing functions into a summation of spherical harmonics. Unfortunately, their approach can not be extended directly to the two dimensional wave equation. Han and Zheng [14, 15] obtained three kinds of exact nonreflecting boundary conditions for exterior problems of wave equation in two and three dimensional space by an approach based on Duhamel's principle. Han and Huang [13] and Han, Yin and Huang [16] derived exact nonreflecting boundary conditions for two and three dimensional Schrödinger equations. Generally speaking, exact boundary conditions require more computational cost.

On the other hand, one can save computational time when using the exact boundary condition by using a fast evolution algorithm. Alpert, Greengard and Hagstrom [1] constructed a fast evolution method for scalar wave equations. Lubich and Schädle [19] constructed the fast convolution method of the nonreflection boundary conditions for wave equations and Schrödinger equations in two dimension.

In this paper, an exact boundary condition for the Klein-Gordon equation in $\mathbb{R}^{2}$ 
and $\mathbb{R}^{3}$ are constructed on the given artificial boundary. Moreover, a series of artificial boundary conditions is derived. With the artificial boundary conditions, the original problem can be reduced to a family of approximate problems on a bounded domain. The stability of the approximate problem is proved, and numerical examples in the $2 \mathrm{D}$ case are given to show the effectiveness of our approach.

\section{Absorbing boundary conditions for the 2D Klein-Gordon equation}

In $\mathbb{R}^{d}$, we define the artificial boundary $\Gamma_{R}$ to be:

$$
\Gamma_{R}=\left\{x|| x \mid=R, R>R_{0}\right\} .
$$

$\Gamma_{R}$ divides $\mathbb{R}^{d}$ into a bounded interior domain $\Omega_{i}=\{x|| x \mid<R\}$ and an unbounded exterior domain $\Omega_{e}=\{x|| x \mid>R\}$. In order to reduce (1.1)-(1.2) into the problem on bounded domain $D_{i}=\Omega_{i} \times[0, T]$, we must study the artificial boundary condition at $\Gamma_{R} \times[0, T]$. In $\mathbb{R}^{2}$, we consider $u(x, t)$, the restriction of the solution of problem (1.1)-(1.2) on $\Omega_{e} \times[0, T] . u(x, t)$ satisfies

$$
\begin{aligned}
\frac{\partial^{2} u}{\partial t^{2}}-\Delta u+u=0, & \forall(x, t) \in \Omega_{e} \times(0, T], \\
\left.u\right|_{\Gamma_{R}}=u(R, \theta, t), & 0 \leq t \leq T, 0 \leq \theta \leq 2 \pi, \\
\left.u\right|_{t=0}=0,\left.u_{t}\right|_{t=0}=0, & R<r<+\infty .
\end{aligned}
$$

Since $\left.u\right|_{\Gamma_{R}}=u(R, \theta, t)$ is unknown, the problem (2.2)-(2.4) is an incompletely posed problem; it can't be solved independently. If $u(R, \theta, t)$ is given, the problem the problem (2.2)-(2.4) is well posed, so the solution $u(r, \theta, t)$ of $(2.2)-(2.4)$ can be given by $u(R, \theta, t)$.

Let the solution of (1.1)-(1.2) satisfy

$$
u(r, \theta, t)=\frac{u_{0}(r, t)}{2}+\sum_{n=1}^{+\infty}\left(u_{n}(r, t) \cos n \theta+v_{n}(r, t) \sin n \theta\right) .
$$

Inserting (2.5) into (2.2) and using (2.3) and (2.4), we obtain $\left\{u_{n}(r, t), n=0,1, \ldots\right\}$ and $\left\{v_{n}(r, t), n=1, \ldots\right\}$, which solve the following problems

$$
\begin{gathered}
\frac{\partial^{2} u_{n}}{\partial t^{2}}-\left(\frac{\partial^{2} u_{n}}{\partial r^{2}}+\frac{1}{r} \frac{\partial u_{n}}{\partial r}-\frac{n^{2}}{r^{2}} u_{n}\right)+u_{n}=0 \quad R<r<+\infty, \quad 0<t \leq T, \\
\left.u_{n}\right|_{r=R}=\alpha_{n}(t), \quad 0<t \leq T, \\
\left.u_{n}\right|_{t=0}=0,\left.\quad\left(u_{n}\right)_{t}\right|_{t=0}=0, \quad R \leq r<+\infty ; \\
\frac{\partial^{2} v_{n}}{\partial t^{2}}-\left(\frac{\partial^{2} v_{n}}{\partial r^{2}}+\frac{1}{r} \frac{\partial v_{n}}{\partial r}-\frac{n^{2}}{r^{2}} v_{n}\right)+v_{n}=0, \quad R<r<+\infty, \quad 0<t \leq T, \\
\left.v_{n}\right|_{r=R}=\beta_{n}(t), \quad 0<t \leq T, \\
\left.v_{n}\right|_{t=0}=0,\left.\quad\left(v_{n}\right)_{t}\right|_{t=0}=0, \quad R \leq r<+\infty ;
\end{gathered}
$$


where

$$
\begin{aligned}
& \alpha_{n}(t)=\frac{1}{\pi} \int_{0}^{2 \pi} u(R, \theta, t) \cos n \theta d \theta, \quad n=0,1, \ldots, \\
& \beta_{n}(t)=\frac{1}{\pi} \int_{0}^{2 \pi} u(R, \theta, t) \sin n \theta d \theta, \quad n=1,2, \ldots
\end{aligned}
$$

In order to solve problems (2.6)-(2.8) and (2.9)-(2.11), firstly, we consider the following auxiliary problem:

$$
\begin{gathered}
\frac{\partial^{2} G_{n}}{\partial t^{2}}-\left(\frac{\partial^{2} G_{n}}{\partial r^{2}}+\frac{1}{r} \frac{\partial G_{n}}{\partial r}-\frac{n^{2}}{r^{2}} G_{n}\right)+G_{n}=0, \quad R<r<+\infty, \quad 0<t \leq T \\
\left.G_{n}\right|_{r=R}=1, \quad 0<t \leq T \\
\left.G_{n}\right|_{t=0}=0,\left.\quad\left(G_{n}\right)_{t}\right|_{t=0}=0, \quad R \leq r<+\infty .
\end{gathered}
$$

Let

$$
G_{n}(r, t)=\sin \left(\left(\omega^{2}+1\right)^{1 / 2} t\right) W(r)
$$

where $\omega>0$ is an arbitrary real number.

Substituting equation (2.17) into (2.14), we find that $W(r)$ satisfies

$$
\frac{d^{2} W}{d r^{2}}+\frac{1}{r} \frac{d W}{d r}+\left(\omega^{2}-\frac{n^{2}}{r^{2}}\right) W=0
$$

The equation (2.18) has two independent solutions: $J_{n}(\omega r), Y_{n}(\omega r)[2]$, where $J_{n}(r)$ and $Y_{n}(r)$ are Bessel functions of the first and second kind, respectively. Thus for all $\omega>0$, functions of the form

$$
\frac{\omega \sin \left(\left(\omega^{2}+1\right)^{1 / 2} t\right)}{\left(\omega^{2}+1\right)^{3 / 2}} \frac{J_{n}(\omega r) Y_{n}(\omega R)-Y_{n}(\omega r) J_{n}(\omega R)}{J_{n}^{2}(\omega R)+Y_{n}^{2}(\omega R)}
$$

are solutions of (2.14).

Define $G_{n}^{*}(r, t)$ to be

$$
G_{n}^{*}(r, t)=\frac{2}{\pi} \int_{0}^{+\infty} \frac{\omega \sin \left(\left(\omega^{2}+1\right)^{1 / 2} t\right)}{\left(\omega^{2}+1\right)^{3 / 2}} \frac{J_{n}(\omega r) Y_{n}(\omega R)-Y_{n}(\omega r) J_{n}(\omega R)}{J_{n}^{2}(\omega R)+Y_{n}^{2}(\omega R)} d \omega
$$

To study the properties of $G_{n}^{*}(r, t)$, we remember the asymptotic behavior of the Bessel functions $J_{n}(z)$ and $Y_{n}(z)$ and their zeros: [9]

$$
\begin{aligned}
J_{n}(z)= & \frac{z^{n}}{2^{n}} \sum_{k=0}^{+\infty}(-1)^{k} \frac{z^{2 k}}{2^{2 k} k ! \Gamma(\nu+k+1)}, \quad(|\arg z|<\pi), \\
\pi Y_{n}(z)= & 2 J_{n}(z)\left(\ln \frac{z}{2}+C\right)-\sum_{k=0}^{n-1} \frac{(n-k-1) !}{k !}\left(\frac{z}{2}\right)^{2 k-n} \\
& -\left(\frac{z}{2}\right)^{n} \frac{1}{n !} \sum_{k=1}^{n} \frac{1}{k}-\sum_{k=1}^{+\infty} \frac{(-1)^{k}\left(\frac{z}{2}\right)^{n+2 k}}{k !(k+n) !}\left[\sum_{m=1}^{n+k} \frac{1}{m}+\sum_{m=1}^{k} \frac{1}{m}\right] .
\end{aligned}
$$


Here, $\mathrm{C}$ is the Euler constant.

From $(2.20)$ and $(2.21)$, there exists a constant $\epsilon \in(0,1)$ such that

$$
\begin{cases}J_{0}(\omega)=1+O\left(\omega^{2}\right), & \omega \rightarrow+0, \\ Y_{0}(\omega)=\frac{2}{\pi}(\ln \omega+C-\ln 2)+O\left(\omega^{2-\epsilon}\right), & \omega \rightarrow+0, \\ J_{n}(\omega)=\frac{1}{n !}\left(\frac{\omega}{2}\right)^{n}+O\left(\omega^{n+2}\right), & \omega \rightarrow+0, \\ Y_{n}(\omega)=-\frac{(n-1) !}{\pi}\left(\frac{\omega}{2}\right)^{-n}+O\left(\omega^{-n+2-\epsilon}\right), & \omega \rightarrow+\infty .\end{cases}
$$

From asymptotic expansion of Hankel functions, we obtain:

$$
\begin{aligned}
J_{n}^{2}(\omega)+Y_{n}^{2}(\omega) & \sim \frac{2}{\pi \omega}\left[\sum_{m=0}^{+\infty}(-1)^{m} \frac{(n, m)}{(2 i \omega)^{m}}\right]\left[\sum_{k=0}^{+\infty} \frac{(n, m)}{(2 i \omega)^{m}}\right] \\
& =\frac{2}{\pi \omega} \sum_{m=0}^{+\infty} \sum_{k=0}^{2 m}(-1)^{k} \frac{(n, k)}{(2 i \omega)^{k}} \frac{(n, 2 m-k)}{(2 i \omega)^{2 m-k}} \\
& =\frac{2}{\pi \omega} \sum_{m=0}^{+\infty} \sum_{k=0}^{2 m}(n, k)(n, 2 m-k) \\
& =\frac{2}{\pi \omega} \sum_{m=0}^{+\infty} \frac{a_{m}^{n}}{\omega^{2 m}}, \quad \omega \rightarrow+\infty .
\end{aligned}
$$

with

$$
\begin{aligned}
(n, k) & =\frac{\Gamma\left(n+\frac{1}{2}+k\right)}{k ! \Gamma\left(n+\frac{1}{2}-k\right)}, \\
a_{m}^{n} & =\frac{1}{(-4)^{m}} \sum_{k=0}^{2 m}(-1)^{k}(n, k)(n, 2 m-k) .
\end{aligned}
$$

Note that $a_{0}^{n}=0$, and let

$$
\frac{1}{\sum_{m=0}^{+\infty} \frac{a_{m}^{n}}{\omega^{2 m}}}=\sum_{m=0}^{+\infty} b_{m}^{n} \frac{1}{\omega^{2 m}}
$$

Then $\left\{b_{m}^{n}\right\}$ can be determined by $\left\{a_{m}^{n}\right\}$,

$$
\begin{aligned}
& b_{0}^{n}=0, \\
& b_{m}^{n} a_{0}^{n}+b_{m-1}^{n} a_{1}^{n}+\cdots+b_{0}^{n} a_{m}^{n}=0,
\end{aligned}
$$

and

$$
\frac{1}{J_{n}^{2}(\omega)+Y_{n}^{2}(\omega)} \sim \frac{\pi \omega}{2}\left[1+\sum_{m=1}^{+\infty} \frac{b_{m}^{n}}{\omega^{2 m}}\right], \quad \omega \rightarrow+\infty .
$$

Thus $G_{n}^{*}(r, t)$ has the following properties: 
1. $G_{n}^{*}(r, t)$ is absolutely integrable on $(r, t) \in[R,+\infty] \times[0, T]$, and $G_{n}^{*}(r, t)$ is continuous in $[R,+\infty] \times[0, T]$.

2. $G_{n}^{*}(r, t)$ is differentiable in $[R,+\infty] \times[0, T] \backslash \Gamma_{R, 0}$, and

$$
\begin{aligned}
& \frac{\partial G_{n}^{*}(r, t)}{\partial r}=\frac{2}{\pi} \int_{0}^{+\infty} \frac{\omega^{2} \sin \left(\left(\omega^{2}+1\right)^{1 / 2} t\right)}{\left(\omega^{2}+1\right)^{3 / 2}} \frac{J_{n}^{\prime}(\omega r) Y_{n}(\omega R)-Y_{n}^{\prime}(\omega r) J_{n}(\omega R)}{J_{n}^{2}(\omega R)+Y_{n}^{2}(\omega R)} d \omega, \\
& \frac{\partial G_{n}^{*}(r, t)}{\partial t}=\frac{2}{\pi} \int_{0}^{+\infty} \frac{\omega \cos \left(\left(\omega^{2}+1\right)^{1 / 2} t\right)}{\omega^{2}+1} \frac{J_{n}(\omega r) Y_{n}(\omega R)-Y_{n}(\omega r) J_{n}(\omega R)}{J_{n}^{2}(\omega R)+Y_{n}^{2}(\omega R)} d \omega,
\end{aligned}
$$

where $\Gamma_{R, 0}$ presents the characteristic $\{(r, t) \mid r=R+t, t \geq 0\}$.

3. $G_{n}^{*}(R, t)=0, G_{n}^{*}(r, 0)=0$.

4. From equality (2.24) and (2.25), we obtain:

$$
\begin{aligned}
\frac{\partial G_{n}^{*}(R, t)}{\partial r} & =-\frac{4}{\pi^{2} R} \int_{0}^{+\infty} \frac{\omega \sin \left(\left(\omega^{2}+1\right)^{1 / 2} t\right)}{\left(\omega^{2}+1\right)^{3 / 2}} \frac{1}{J_{n}^{2}(\omega R)+Y_{n}^{2}(\omega R)} d \omega, t>0 \\
\frac{\partial G_{n}^{*}(r, 0)}{\partial t} & =\frac{2}{\pi} \int_{0}^{+\infty} \frac{w}{\omega^{2}+1} \frac{J_{n}(\omega r) Y_{n}(\omega R)-Y_{n}(\omega r) J_{n}(\omega R)}{J_{n}^{2}(\omega R)+Y_{n}^{2}(\omega R)} d \omega \\
& =-\frac{K_{n}(r)}{K_{n}(R)}, \quad r>R .
\end{aligned}
$$

The last equality is proved in the appendix. It will play an important role in this section.

5. $G_{n}^{*}(r, t)$ is a weak solution of $(2.14)$.

Note that $t \frac{K_{n}(r)}{K_{n}(R)}$ is also a solution of (2.14), and let

$$
G_{n}(r, t)=t \frac{K_{n}(r)}{K_{n}(R)}+G_{n}^{*}(r, t) .
$$

Then $G_{n}(r, t)$ is a solution of the initial-boundary value problem (2.14)-(2.16).

Using Duhamel's Theorem, one can derive $u_{n}(r, t)$, which is the solution to $(2.6)-$ (2.8), and $v_{n}(r, t)$, which is the solution to (2.9)-(2.11), from $G_{n}(r, t)$ :

$$
\begin{aligned}
u_{n}(r, t) & =\int_{0}^{t} \frac{\partial \alpha_{n}(\tau)}{\partial \tau} \frac{\partial G_{n}(r, t-\tau)}{\partial t} d \tau \\
& =\frac{K_{n}(r)}{K_{n}(R)} \alpha_{n}(t)+\int_{0}^{t} \frac{\partial^{2} \alpha_{n}(\tau)}{\partial \tau^{2}} G_{n}^{*}(r, t-\tau) d \tau \\
v_{n}(r, t) & =\int_{0}^{t} \frac{\partial \beta_{n}(\tau)}{\partial \tau} \frac{\partial G_{n}(r, t-\tau)}{\partial t} d \tau \\
& =\frac{K_{n}(r)}{K_{n}(R)} \beta_{n}(t)+\int_{0}^{t} \frac{\partial^{2} \beta_{n}(\tau)}{\partial \tau^{2}} G_{n}^{*}(r, t-\tau) d \tau .
\end{aligned}
$$


On the boundary $r=R$

$$
\begin{aligned}
\frac{\partial u_{n}(R, t)}{\partial r} & =\frac{K_{n}^{\prime}(R)}{K_{n}(R)} u_{n}(R, t)+\int_{0}^{t} \frac{\partial^{2} \alpha_{n}(\tau)}{\partial \tau^{2}} \frac{\partial G_{n}^{*}(R, t-\tau)}{\partial r} d \tau \\
& =\frac{K_{n}^{\prime}(R)}{K_{n}(R)} u_{n}(R, t)+\int_{0}^{t} \frac{\partial^{2} u_{n}(R, \tau)}{\partial \tau^{2}} \frac{\partial G_{n}^{*}(R, t-\tau)}{\partial r} d \tau, \\
\frac{\partial v_{n}(R, t)}{\partial r} & =\frac{K_{n}^{\prime}(R)}{K_{n}(R)} v_{n}(R, t)+\int_{0}^{t} \frac{\partial^{2} v_{n}(R, \tau)}{\partial \tau^{2}} \frac{\partial G_{n}^{*}(R, t-\tau)}{\partial R} d \tau .
\end{aligned}
$$

On the other hand,

$$
\begin{aligned}
\frac{\partial G_{n}^{*}(R, t)}{\partial r} & =-\frac{4}{R \pi^{2}} \int_{0}^{+\infty} \frac{\omega \sin \left(\left(\omega^{2}+1\right)^{1 / 2} t\right)}{\left(\omega^{2}+1\right)^{3 / 2}} \frac{1}{J_{n}^{2}(\omega R)+Y_{n}^{2}(\omega R)} d \omega \\
& \equiv-H Y_{n, R}(t),
\end{aligned}
$$

with

$$
H Y_{n, R}(t)=\frac{4}{R \pi^{2}} \int_{0}^{+\infty} \frac{\omega \sin \left(\left(\omega^{2}+1\right)^{1 / 2} t\right)}{\left(\omega^{2}+1\right)^{3 / 2}} \frac{1}{J_{n}^{2}(\omega R)+Y_{n}^{2}(\omega R)} d \omega .
$$

For arbitrary $t>0$, the integral in (2.32) is convergent, thus (2.32) defines new special functions $H Y_{n, R}(t) . H Y_{n, R}(t)$ can be rewritten into the following equivalent formula:

$$
\begin{aligned}
H Y_{n, R}(t)= & \frac{4}{R \pi^{2}} \int_{0}^{+\infty \omega \sin \left(\left(\omega^{2}+1\right)^{\frac{1}{2}} t\right)} \frac{1}{\left(\omega^{2}+1\right)^{\frac{3}{2}}}\left\{\frac{\pi}{J_{n}^{2}(\omega R)+Y_{n}^{2}(\omega R)}-\frac{\pi}{2} R\left(\omega^{2}+1\right)^{\frac{1}{2}}\right\} d \omega \\
& +\frac{2}{\pi} \int_{0}^{+\infty} \frac{\omega \sin \left(\left(\omega^{2}+1\right)^{1 / 2} t\right)}{\omega^{2}+1} d \omega .
\end{aligned}
$$

Since

$$
\begin{aligned}
\frac{2}{\pi} \int_{0}^{+\infty} \frac{\omega \sin \left(\left(\omega^{2}+1\right)^{1 / 2} t\right)}{\omega^{2}+1} d \omega & =\frac{2}{\pi} \int_{1}^{+\infty} \frac{\sin \xi t}{\xi} d \xi \\
& =\frac{2}{\pi}\left(\int_{0}^{+\infty} \frac{\sin \xi t}{\xi} d \xi-\int_{0}^{1} \frac{\sin \xi t}{\xi} d \xi\right) \\
& =1-\frac{2}{\pi} \int_{0}^{t} \frac{\sin \xi}{\xi} d \xi
\end{aligned}
$$

then if we combine (2.33)-(2.34), we obtain

$$
\begin{aligned}
H Y_{n, R}(t)= & \frac{4}{R \pi^{2}} \int_{0}^{+\infty} \frac{\omega \sin \left(\left(\omega^{2}+1\right)^{1 / 2} t\right)}{\left(\omega^{2}+1\right)^{3 / 2}}\left\{\frac{1}{J_{n}^{2}(\omega R)+Y_{n}^{2}(\omega R)}-\frac{\pi}{2} R\left(\omega^{2}+1\right)^{1 / 2}\right\} d \omega \\
& +1-\frac{2}{\pi} \int_{0}^{t} \frac{\sin \xi}{\xi} d \xi .
\end{aligned}
$$

From (2.35), $H Y_{n, R}(t)$ is differentiable with respect to $t$ in $(0,+\infty)$, and

$$
\begin{aligned}
H Y_{n, R}^{\prime}(t)= & \frac{4}{R \pi^{2}} \int_{0}^{+\infty} \frac{\omega \cos \left(\left(\omega^{2}+1\right)^{1 / 2} t\right)}{\omega^{2}+1}\left\{\frac{1}{J_{n}^{2}(\omega R)+Y_{n}^{2}(\omega R)}-\frac{\pi}{2} R\left(\omega^{2}+1\right)^{1 / 2}\right\} \\
& -\frac{2}{\pi}\left(\frac{\sin t}{t}-1\right) .
\end{aligned}
$$


We can use (2.35)-(2.36) to calculate $H Y_{n, R}(t)$ and $H Y_{n, R}^{\prime}(t)$. Substituting (2.31) into (2.29), we have

$$
\begin{aligned}
\frac{\partial u_{n}(R, t)}{\partial r} & =\frac{K_{n}^{\prime}(R)}{K_{n}(R)} u_{n}(R, t)-\int_{0}^{t} \frac{\partial^{2} u_{n}(R, \tau)}{\partial \tau^{2}} H Y_{n, R}(t-\tau) d \tau \\
& =\frac{K_{n}^{\prime}(R)}{K_{n}(R)} u_{n}(R, t)-\frac{\partial u_{n}(R, t)}{\partial t}-\int_{0}^{t} \frac{\partial u_{n}(R, \tau)}{\partial \tau} H Y_{n, R}^{\prime}(t-\tau) d \tau .
\end{aligned}
$$

Similarly, for $v_{n}(r, t)$, we obtain

$$
\frac{\partial v_{n}(R, t)}{\partial r}=\frac{K_{n}^{\prime}(R)}{K_{n}(R)} v_{n}(R, t)-\frac{\partial v_{n}(R, t)}{\partial t}-\int_{0}^{t} \frac{\partial v_{n}(R, \tau)}{\partial \tau} H Y_{n, R}^{\prime}(t-\tau) d \tau .
$$

Equations (2.37)-(2.38) are the exact boundary conditions for $u_{n}(r, t)$ and $v_{n}(r, t)$ on the artificial boundary $\Gamma_{R} \times[0, T]$.

From (2.5), (2.37) and (2.38), we derive the absorbing boundary condition satisfying the solution $u(r, t)$ of Klein-Gordon equation(1.1)-(1.2) on the artificial boundary $\Gamma_{R} \times[0, T]:$

$$
\begin{aligned}
\frac{\partial u(R, \theta, t)}{\partial r}= & -\frac{\partial u(R, \theta, t)}{\partial t}+\sum_{n=0}^{+\infty}\left\{\frac{K_{n}^{\prime}(R)}{K_{n}(R)}\left(u_{n}(R, t) \cos n \theta+v_{n}(R, t) \sin n \theta\right)\right. \\
& \left.-\int_{0}^{t}\left(\frac{\partial u_{n}(R, \tau)}{\partial \tau} \cos n \theta+\frac{\partial v_{n}(R, \tau)}{\partial \tau} \sin n \theta\right) H Y_{n, R}^{\prime}(t-\tau) d \tau\right\}, \\
= & -\frac{\partial u(R, \theta, t)}{\partial t}+\sum_{n=0}^{+\infty}\left\{\frac{K_{n}^{\prime}(R)}{\pi K_{n}(R)} \int_{0}^{2 \pi} u(R, \varphi, t) \cos n(\varphi-\theta) d \varphi\right\} \\
& -\frac{1}{\pi} \int_{0}^{t} \int_{0}^{2 \pi} \frac{\partial u(R, \varphi, \tau)}{\partial \tau} \cos n(\varphi-\theta) H Y_{n, R}^{\prime}(t-\tau) d \tau \\
\equiv & \mathcal{K}^{2}(u(R, \theta, t)) .
\end{aligned}
$$

Therefore, the original problem is transformed into the following initial-boundary value problem on the bounded domain $D_{i}^{T}=D_{i} \times[0, T]$.

$$
\begin{gathered}
\frac{\partial^{2} u}{\partial t^{2}}=\Delta u+u, \quad \forall(x, t) \in \Omega_{i}^{T}, \\
\left.\frac{\partial u}{\partial r}\right|_{\Gamma_{R}}=\mathcal{K}^{2}(u(R, \theta, t)), \quad 0 \leq t \leq T, 0 \leq \theta \leq 2 \pi, \\
\left.u\right|_{t=0}=0,\left.u_{t}\right|_{t=0}=0, \quad R<r<+\infty .
\end{gathered}
$$

If we take the first few terms of the above summation, namely $N=0,1,2, \ldots$,

$$
\begin{aligned}
\frac{\partial u(R, \theta, t)}{\partial r}= & -\frac{1}{\pi} \sum_{n=0}^{N} \int_{0}^{2 \pi} \frac{\partial u(R, \varphi, t)}{\partial t} \cos n(\varphi-\theta) d \varphi \\
& +\frac{1}{\pi} \sum_{n=0}^{N}\left\{\frac{K_{n}^{\prime}(R)}{K_{n}(R)} \int_{0}^{2 \pi} u(R, \varphi, t) \cos n(\varphi-\theta) d \varphi\right. \\
& \left.-\int_{0}^{t} \int_{0}^{2 \pi} \frac{\partial u(R, \varphi, \tau)}{\partial \tau} \cos n(\varphi-\theta) H Y_{n, R}^{\prime}(t-\tau) d \varphi d \tau\right\} \\
\equiv & \mathcal{K}_{N}^{2}(u(R, \theta, t)) .
\end{aligned}
$$


If we use boundary condition (2.40) instead of (2.39), we obtain a series of approximate problems.

3. Absorbing boundary conditions for the 3D Klein-Gordon equation

When $d=3$, consider the restriction of the solution $\mathcal{U}$ of (1.1)-(1.2) on $\Omega_{e} \times[0, T]$, which satisfyies the following equations in spherical coordinates:

$$
\begin{gathered}
\frac{\partial^{2} \mathcal{U}}{\partial t^{2}}-\left\{\frac{\partial^{2} \mathcal{U}}{\partial r^{2}}+\frac{2}{r} \frac{\partial \mathcal{U}}{\partial r}+\frac{1}{r^{2} \sin \theta}\left(\sin \theta \frac{\partial \mathcal{U}}{\partial \theta}+\frac{1}{r^{2} \sin ^{2} \theta} \frac{\partial^{2} \mathcal{U}}{\partial \varphi^{2}}\right)\right\}+\mathcal{U}=0 \\
\quad R<r,-\frac{\pi}{2} \leq \theta \leq \frac{\pi}{2}, 0 \leq \varphi \leq 2 \pi \\
\left.\mathcal{U}\right|_{\Gamma_{R}}=\mathcal{U}(R, \theta, \varphi, t), \quad 0 \leq t \leq T, \\
\left.\mathcal{U}\right|_{t=0}=0,\left.\quad \mathcal{U}_{t}\right|_{t=0}=0, \quad R<r<+\infty .
\end{gathered}
$$

If $\mathcal{U}(R, \theta, \varphi, t)$ is known, problem (3.1)-(3.3) can be solved uniquely. Giving the expansions of the solution of (3.1)-(3.3) in spherical harmonic functions $\left\{Y_{n}^{m}(\theta, \varphi), n \geq\right.$ $0,-n \leq m \leq n\}$, namely,

$$
\mathcal{U}(r, \theta, \varphi, t)=\sum_{n=0}^{+\infty} \sum_{m=-n}^{n} \mathcal{U}_{n}^{m}(r, t) Y_{n}^{m}(\theta, \varphi)
$$

where

$$
\mathcal{U}_{n}^{m}(r, t)=\int_{\Gamma_{1}} \mathcal{U}(r, \theta, \varphi, t) \bar{Y}_{n}^{m}(\theta, \varphi) d s
$$

where $\Gamma_{1}$ is the surface of the unit ball.

By substituting (3.4) into (3.1) and using the initial and boundary value conditions (3.2)-(3.3), one can show that $\left\{\mathcal{U}_{n}^{m}(r, t), n \geq 0,-n \leq m \leq n\right\}$ satisfies

$$
\begin{gathered}
\frac{\partial^{2} \mathcal{U}_{n}^{m}}{\partial t^{2}}-\left\{\frac{\partial \mathcal{U}_{n}^{m}}{\partial r^{2}}+\frac{2}{r} \frac{\partial \mathcal{U}_{n}^{m}}{\partial r}-\frac{n(n+1)}{r^{2}} \mathcal{U}_{n}^{m}\right\}+\mathcal{U}_{n}^{m}=0, \\
\quad \forall(r, t) \in(R,+\infty) \times(0, T] . \\
\left.\mathcal{U}_{n}^{m}\right|_{r=R}=\mathcal{U}_{n}^{m}(R, t), \quad 0 \leq t \leq T, \\
\left.\mathcal{U}_{n}^{m}\right|_{t=0}=0,\left.\quad\left(\mathcal{U}_{n}^{m}\right)\right|_{t=0}=0, \quad R<r<+\infty .
\end{gathered}
$$

In order to find the solution of problem (3.6)-(3.8), we consider the following auxiliary problem:

$$
\begin{gathered}
\frac{\partial^{2} H_{n}}{\partial t^{2}}-\left\{\frac{\partial H_{n}}{\partial r^{2}}+\frac{2}{r} \frac{\partial H_{n}}{\partial r}-\frac{n(n+1)}{r^{2}} H_{n}\right\}+H_{n}=0, \\
\forall(r, t) \in(R,+\infty) \times(0, T] . \\
\left.\frac{\partial H_{n}}{\partial t}\right|_{r=R}=1, \quad 0 \leq t \leq T, \\
\left.H_{n}\right|_{t=0}=0,\left.\quad\left(H_{n}\right)_{t}\right|_{t=0}=0, \quad R<r<+\infty .
\end{gathered}
$$


$\forall \omega>0$, assume $\sin \left(\left(\omega^{2}+1\right)^{1 / 2} t\right) W(r)$ is a solution of $(3.9)$. Then $W(r)$ satisfies

$$
\frac{d^{2} W}{d r^{2}}+\frac{2}{r} \frac{d W}{d r}+\left(\omega^{2}-\frac{n(n+1)}{r^{2}}\right) W=0
$$

Equation (3.12) is spherical Bessel equation; it has two independent solutions $h_{1}(\omega r)$ and $h_{2}(\omega r)$, where

$$
h_{1}(\rho)=\sqrt{\frac{\pi}{2 \rho}} J_{n+1 / 2}(\rho), \quad h_{2}(\rho)=\sqrt{\frac{\pi}{2 \rho}} Y_{n+1 / 2}(\rho) .
$$

Let

$$
H_{n}^{*}(r, t)=\frac{2}{\pi} \int_{0}^{+\infty} \frac{\omega \sin \left(\left(\omega^{2}+1\right)^{1 / 2} t\right)}{\left(\omega^{2}+1\right)^{3 / 2}} \frac{h_{1}(\omega r) h_{2}(\omega R)-h_{1}(\omega R) h_{1}(\omega r)}{h_{1}^{2}(\omega R)+h_{2}^{2}(\omega R)} d \omega
$$

For $(r, t) \in[R,+\infty) \times[0, T]$, the integral in 3.14 is convergent. $H_{n}^{*}(r, t)$ is a solution of (3.9), and

$$
\begin{aligned}
H_{n}^{*}(R, t) & =0, \quad 0 \leq t \leq T \\
H_{n}^{*}(r, 0) & =0, \quad R \leq r<+\infty . \\
\frac{\partial H_{n}^{*}(r, 0)}{\partial t} & =\frac{2}{\pi} \int_{0}^{+\infty} \frac{\omega}{\omega^{2}+1} \frac{h_{1}(\omega r) h_{2}(\omega R)-h_{1}(\omega R) h_{1}(\omega r)}{h_{1}^{2}(\omega R)+h_{2}^{2}(\omega R)} d \omega \\
& =\frac{2}{\pi} \sqrt{\frac{R}{r}} \int_{0}^{+\infty} \frac{\omega}{\omega^{2}+1} \frac{J_{n+1 / 2}(\omega r) Y_{n+1 / 2}(\omega R)-J_{n+1 / 2}(\omega R) Y_{n+1 / 2}(\omega r)}{J_{n+1 / 2}^{2}(\omega R)+Y_{n+1 / 2}^{2}(\omega R)} d \omega \\
& =-\sqrt{\frac{R}{r}} \frac{K_{n+1 / 2}(r)}{K_{n+1 / 2}(R)},
\end{aligned}
$$

where the last equality is obtained from (A.3) in the appendix.

Let

$$
L_{n+1 / 2}(r)=\frac{K_{n+1 / 2}(r)}{\sqrt{r}}
$$

Then $L_{n+1 / 2}(r)$ satisfies the following differential equation:

$$
\frac{d^{2} L_{n+1 / 2}}{d r^{2}}+\frac{2}{r} \frac{d L_{n+1 / 2}}{d r}-\left(1+\frac{n(n+1)}{r^{2}}\right) L_{n+1 / 2}=0
$$

Thus $L_{n+1 / 2}(r)$ is a special solution of (3.9), and

$$
\frac{\partial H_{n}^{*}(r, 0)}{\partial t}=-\frac{L_{n+1 / 2}(r)}{L_{n+1 / 2}(R)}
$$

Let

$$
H_{n}(r, t)=t \frac{L_{n+1 / 2}(r)}{L_{n+1 / 2}(R)}+H_{n}^{*}(r, t) .
$$


Then $H_{n}(r, t)$ is a solution of the initial-boundary value problem (3.9)-(3.11). Using Duhamel's theorem and $H_{n}(r, t)$, we can find the solution $\mathcal{U}_{n}^{m}(r, t)$ of $(3.6)-(3.8)$ :

$$
\begin{aligned}
\mathcal{U}_{n}^{m}(r, t) & =\int_{0}^{t} \frac{\partial \mathcal{U}_{n}^{m}(R, \tau)}{\partial \tau} \frac{\partial H_{n}(r, t-\tau)}{\partial t} d \tau \\
& =\frac{L_{n+1 / 2}(r)}{L_{n+1 / 2}(R)} \mathcal{U}_{n}^{m}(R, t)+\int_{0}^{t} \frac{\partial^{2} \mathcal{U}_{n}^{m}(R, \tau)}{\partial \tau^{2}} H_{n}^{*}(r, t-\tau) d \tau
\end{aligned}
$$

On the boundary $r=R$,

$$
\frac{\partial \mathcal{U}_{n}^{m}(R, t)}{\partial r}=\frac{L_{n+1 / 2}^{\prime}(R)}{L_{n+1 / 2}(R)} \mathcal{U}_{n}^{m}(R, t)+\int_{0}^{t} \frac{\partial^{2} \mathcal{U}_{n}^{m}(R, \tau)}{\partial \tau^{2}} H_{n}^{*}(R, t-\tau) d \tau
$$

On the other hand

$$
\begin{aligned}
\frac{\partial H_{n}^{*}(R, t)}{\partial r} & =\frac{2}{\pi} \int_{0}^{+\infty} \frac{\omega^{2} \sin \left(\left(\omega^{2}+1\right)^{1 / 2} t\right)}{\left(\omega^{2}+1\right)^{3 / 2}} \\
& \times \frac{J_{n+\frac{1}{2}}^{\prime}(\omega R) Y_{n+\frac{1}{2}}(\omega R)-J_{n+\frac{1}{2}}(\omega R) Y_{n+\frac{1}{2}}^{\prime}(\omega R)}{J_{n+\frac{1}{2}}^{2}(\omega R)+Y_{n+\frac{1}{2}}^{2}(\omega R)} d \omega \\
& =-\frac{4}{\pi^{2} R} \int_{0}^{+\infty} \frac{\omega \sin \left(\left(\omega^{2}+1\right)^{1 / 2} t\right)}{\omega^{2}+1} \frac{1}{J_{n+\frac{1}{2}}^{2}(\omega R)+Y_{n+\frac{1}{2}}^{2}(\omega R)} d \omega \\
& \equiv-H Y_{n+1 / 2, R}(t) .
\end{aligned}
$$

From (3.18), we obtain

$$
\begin{aligned}
\frac{\partial \mathcal{U}_{n}^{m}(R, t)}{\partial r} & =\frac{L_{n+1 / 2}^{\prime}(R)}{L_{n+1 / 2}(R)} \mathcal{U}_{n}^{m}(R, t)-\int_{0}^{t} \frac{\partial^{2} \mathcal{U}_{n}^{m}(R, \tau)}{\partial \tau^{2}} H Y_{n+1 / 2, R}(t-\tau) d \tau \\
& =\frac{L_{n+\frac{1}{2}}^{\prime}(R)}{L_{n+\frac{1}{2}}(R)} \mathcal{U}_{n}^{m}(R, t)-\int_{0}^{t} \frac{\partial \mathcal{U}_{n}^{m}(R, \tau)}{\partial \tau} H Y_{n+\frac{1}{2}, R}^{\prime}(t-\tau) d \tau
\end{aligned}
$$

Combining (3.4), (3.5) and (3.19), we get the absorbing boundary condition for the Klein-Gordon equation:

$$
\begin{aligned}
\frac{\partial \mathcal{U}(r, \theta, \varphi, t)}{\partial r}= & \sum_{n=0}^{+\infty} \sum_{m=-n}^{n}\left\{\frac{L_{n+1 / 2}^{\prime}(R)}{L_{n+1 / 2}(R)} \int_{S_{1}} \mathcal{U}\left(R, \theta^{\prime}, \varphi^{\prime}, t\right) \bar{Y}_{n}^{m}\left(\theta^{\prime}, \varphi^{\prime}\right) d S^{\prime}\right. \\
& -\int_{0}^{t} \int_{S_{1}} \frac{\partial \mathcal{U}\left(R, \theta^{\prime}, \varphi^{\prime}, \tau\right)}{\partial \tau} H Y_{n+1 / 2, R}(t-\tau) d S^{\prime} \\
& \left.-\int_{S_{1}} \frac{\partial \mathcal{U}\left(R, \theta^{\prime}, \varphi^{\prime}, t\right)}{\partial t} \bar{Y}_{n}^{m}\left(\theta^{\prime}, \varphi^{\prime}\right) d S^{\prime}\right\} Y_{n}^{m}(\theta, \varphi) \\
\equiv & \mathcal{K}^{3}(\mathcal{U}(r, \theta, \varphi, t)) .
\end{aligned}
$$

Taking $N$ terms in the right hand side of (3.20), we obtain a series of approximate 
artificial boundary conditions:

$$
\begin{aligned}
\frac{\partial \mathcal{U}(r, \theta, \varphi, t)}{\partial r}= & \sum_{n=0}^{N} \sum_{m=-n}^{n}\left\{\frac{L_{n+1 / 2}^{\prime}(R)}{L_{n+1 / 2}(R)} \int_{S_{1}} \mathcal{U}\left(R, \theta^{\prime}, \varphi^{\prime}, t\right) \bar{Y}_{n}^{m}\left(\theta^{\prime}, \varphi^{\prime}\right) d S^{\prime}\right. \\
& -\int_{0}^{t} \int_{S_{1}} \frac{\partial \mathcal{U}\left(R, \theta^{\prime}, \varphi^{\prime}, \tau\right)}{\partial \tau} H Y_{n+1 / 2, R}(t-\tau) d S^{\prime} \\
& \left.-\int_{S_{1}} \frac{\partial \mathcal{U}\left(R, \theta^{\prime}, \varphi^{\prime}, t\right)}{\partial t} \bar{Y}_{n}^{m}\left(\theta^{\prime}, \varphi^{\prime}\right) d S^{\prime}\right\} Y_{n}^{m}(\theta, \varphi) \\
\equiv & \mathcal{K}_{N}^{3}(\mathcal{U}(r, \theta, \varphi, t)) .
\end{aligned}
$$

4. Stability analysis of the reduced problems on the bounded computational domain $D_{i}$

By using the absorbing boundary conditions (2.39) and (3.20), the original problem (1.1)-(1.2) on the unbounded domain $\mathbb{R}^{d} \times[0, T]$ is transformed into the following initial-boundary value problem on the bounded domain $D_{i}^{T}=D_{i} \times[0, T]$, for $d=2,3$,

$$
\begin{aligned}
& \frac{\partial^{2} u}{\partial t^{2}}-\Delta u+u=f(x, t), \forall(x, t) \in D_{i}^{T}, \\
&\left.u\right|_{t=0}=\varphi_{0}(x),\left.\quad u_{t}\right|_{t=0}=\varphi_{1}(x), \quad \forall x \in \Omega_{i}, \\
&\left.\frac{\partial u}{\partial r}\right|_{\Gamma_{R}}=\mathcal{K}^{d}\left(\left.u\right|_{\Gamma_{R}}\right), \quad \forall t \in[0, T] .
\end{aligned}
$$

Similarly, by using approximate boundary conditions (2.40) and (3.21), we can reduce the original problem to an approximate initial-boundary value problem on the bounded computational domain $D_{i}^{T}$,

$$
\begin{aligned}
& \frac{\partial^{2} u^{N}}{\partial t^{2}}-\Delta u^{N}+u^{N}=f(x, t), \quad \forall(x, t) \in D_{i}^{T}, \\
\left.u^{N}\right|_{t=0}=\varphi_{0}(x),\left.\quad\left(u^{N}\right)_{t}\right|_{t=0}=\varphi_{1}(x), & \forall x \in \Omega_{i}, \\
\left.\frac{\partial u^{N}}{\partial r}\right|_{\Gamma_{R}}=\mathcal{K}_{N}^{d}\left(\left.u^{N}\right|_{\Gamma_{R}}\right), & \forall t \in[0, T] .
\end{aligned}
$$

We have the following stability estimate:

THEOREM 4.1. The initial-boundary value problem (4.1)-(4.3) ((4.4)-(4.6)) on the bounded the computational domain $D_{i}^{T}$ has at most one solution $u(x, t)\left(u^{N}(x, t)\right)$, and $u(x, t)\left(u^{N}(x, t)\right)$ depends continuously on the initial values $\varphi_{0}(x), \varphi_{1}(x)$ and $f(x, t)$.

Proof. We only prove the results in two dimensional case, but the three dimensional case can be proved similarly.

By multiplying both sides of $(4.1)$ by $\frac{\partial u(x, t)}{\partial t}$, integrating on $\Omega_{i}$, and using the initial conditions (4.5) and boundary condition (4.6), we obtain

$$
\frac{1}{2} \frac{\mathrm{d}}{\mathrm{d} t}\left\{\int_{\Omega_{i}}\left[\left|\frac{\partial u}{\partial t}\right|^{2}+|\nabla u|^{2}+u^{2}\right] \mathrm{d} x\right\}-\left.\left.\frac{\partial u}{\partial x}\right|_{\Gamma_{R}} \frac{\partial u}{\partial t}\right|_{\Gamma_{R}}=\int_{\Omega_{i}} f \frac{\partial u}{\partial t} \mathrm{~d} x .
$$

In order to evaluate the term $\left.\left.\frac{\partial u}{\partial x}\right|_{\Gamma_{R}} \frac{\partial u}{\partial t}\right|_{\Gamma_{R}}$, we consider the following auxiliary problem: 


$$
\begin{aligned}
\frac{\partial^{2} w}{\partial t^{2}}-\Delta w+w=0, & \forall(x, t) \in \Omega_{e} \times(0, T], \\
\left.w\right|_{\Gamma_{R}}=\left.u\right|_{\Gamma_{R}}, & 0 \leq t \leq T, \\
\left.w\right|_{t=0}=0,\left.w_{t}\right|_{t=0}=0, & |x|>R .
\end{aligned}
$$

From (2.39), we have

$$
\left.\frac{\partial w}{\partial x}\right|_{\Gamma_{R}}=\left.\frac{\partial u}{\partial x}\right|_{\Gamma_{R}}=\mathcal{K}^{2}\left(\left.u\right|_{\Gamma_{R}},\left.\frac{\partial u}{\partial t}\right|_{\Gamma_{R}}\right) .
$$

Multiplying both sides of (4.8) by $\frac{\partial w}{\partial t}$ and integrating on $\Omega_{e}$, we arrive at

$$
\left.\left.\frac{\partial u}{\partial x}\right|_{\Gamma_{R}} \frac{\partial u}{\partial t}\right|_{\Gamma_{R}}=\frac{1}{2}\left\{\int_{\Omega_{e}}\left[\left|\frac{\partial w}{\partial t}\right|^{2}+|\nabla w|^{2}+w^{2}\right] d x\right\} .
$$

Combining (4.7) and (4.11), we obtain

$$
\frac{d}{d t} E(t) \leq E(t)+F(t), 0 \leq t \leq T,
$$

where

$$
\begin{aligned}
& E(t)=\frac{1}{2} \int_{\Omega_{i}}\left[\left|\frac{\partial u}{\partial t}\right|^{2}+|\nabla u|^{2}+u^{2}\right] \mathrm{d} x+\frac{1}{2} \int_{\Omega_{e}}\left[\left|\frac{\partial w}{\partial t}\right|^{2}+|\nabla w|^{2}+w^{2}\right] d x, \\
& F(t)=\int_{\Omega_{i}}|f(x, t)|^{2} d x .
\end{aligned}
$$

Using Gronwall's inequality, we arrive at

$$
E(t) \leq e^{t}(E(0)+F(t)) .
$$

On the other hand

$$
E(0)=\frac{1}{2} \int_{\Omega_{i}}\left[\left|\varphi_{1}(x)\right|^{2}+\left|\varphi_{0}^{\prime}(x)\right|^{2}+\left|\varphi_{0}(x)\right|^{2}\right] d x .
$$

From (4.13), we have a stability estimate for the solution $u(x, t)$ :

$$
\begin{aligned}
& \frac{1}{2} \int_{\Omega_{i}}\left[\left|\frac{\partial u(x, t)}{\partial t}\right|^{2}+|\nabla u(x, t)|^{2}+|u(x, t)|^{2}\right] \mathrm{d} x \\
\leq & e^{t}\left\{\frac{1}{2} \int_{\Omega_{i}}\left[\left|\varphi_{1}(x)\right|^{2}+\left|\varphi_{0}^{\prime}(x)\right|^{2}+\left|\varphi_{0}(x)\right|^{2}\right] d x+\int_{\Omega_{i}}|f(x, t)|^{2} d x\right\} .
\end{aligned}
$$

The statement for $u^{N}(x, t)$ can be proved similarly.

5. The kernel functions $\left\{H Y_{n, R}^{\prime}(t), n=0,1,2, \ldots\right\}$

The kernel functions $\left\{H Y_{n, R}^{\prime}(t), n=0,1,2, \ldots\right\}$ are very important in the absorbing boundary condition (2.39). Before discussing the numerical solution of problem (4.4)-(4.6) we must calculate the functions $\left\{H Y_{n, R}^{\prime}(t), n=0,1,2, \ldots\right\}$. The integrals possess at most two singular points 0 and $+\infty$. But for large $t>0$, the integrals become oscillatory. We will use a scheme similar to the one found in [14] to get their approximate values when the parameter is not too large.

In practical computation, we introduce a large number $M$ to divide the semiinfinite interval $(0,+\infty)$ into two parts: $(0, M)$ and $(M,+\infty)$. First, consider the first integral in $(2.35)$ on $(M,+\infty)$. By the asymptotic expansion (2.23), it holds 


$$
\begin{aligned}
\frac{4}{\pi^{2}} \int_{M}^{+\infty} \frac{\omega \cos \left(\left(\omega^{2}+1\right)^{1 / 2} t\right)}{\omega^{2}+1} \frac{1}{J_{n}^{2}(\omega)+Y_{n}^{2}(\omega)} d w & \approx \frac{2}{\pi} \sum_{m=0}^{+\infty} b_{m}^{n} \int_{M}^{+\infty} \frac{\cos (w t)}{w^{2 m+1}} d w \\
& \equiv \frac{2}{\pi} \sum_{m=0}^{\infty} b_{m}^{n} S_{M}(m, t)
\end{aligned}
$$

where

$$
\begin{aligned}
S_{M}(m, t) & =\int_{M}^{+\infty} \frac{\cos (w t)}{w^{2 m+1}} d w \\
& =\frac{\cos (M t)}{2 m M^{2 m}}-\frac{t \cos (M t)}{2 m(2 m-1) M^{2 m-1}}+\frac{t^{2}}{2 m(2 m-1)} S_{M}(m-1, t), \\
S_{M}(0, t) & =\int_{M}^{+\infty} \frac{\cos (w t)}{w} d w=\int_{M_{t}}^{+\infty} \frac{\cos (w)}{w}=\frac{\pi}{2}-\int_{0}^{M_{t}} \frac{\cos w}{w} d w .
\end{aligned}
$$

The function $S_{M}(0, t)$ can be obtained by numerical quadrature for any fixed $M$ and $t$. Secondly, consider the integral on $(0, M)$, namely,

$$
\frac{4}{\pi^{2}} \int_{0}^{M} \frac{\omega \cos \left(\left(\omega^{2}+1\right)^{1 / 2} t\right)}{\omega^{2}+1} \frac{1}{J_{n}^{2}(\omega)+Y_{n}^{2}(\omega)} d w .
$$

The Simpson quadrature scheme can be applied to calculate this integral.

From Figures 5.1-5.2, we can see that $\left\{H Y_{n, R}^{\prime}(t), n=0,1,2, \ldots\right\}$ are smooth and decay very fast. After discretizing the boundary condition (2.40), we need to calculate the summation

$$
\sum_{k=1}^{l} u\left(R, ., t_{l-k}\right) C_{n}(k)
$$

with $t_{k}=k \Delta t, k=1, \cdots, l$ and

$$
C_{n}(k)=\int_{t_{k-1}}^{t_{k}} H Y_{n, R}^{\prime}(\lambda) d \lambda-\int_{t_{k}}^{t_{k+1}} H Y_{n, R}^{\prime}(\lambda) d \lambda
$$

As $k \rightarrow \infty, C_{n}(k)$ goes like $O\left(\left(\frac{1}{k}+\Delta t\right) \sqrt{\frac{\Delta t}{k}}\right)$ (see FIG. 5.3 )

\section{Numerical examples}

In this section, we perform numerical examples for the 2D Klein-Gordon equation.

EXAMPle 6.1. First we consider a simple example:

$$
\begin{array}{r}
\frac{\partial^{2}}{\partial t^{2}} u(x, y, t)-\Delta u(x, y, t)+u(x, y, t)=0, \\
\left.u(x, y, t)\right|_{t=0}=g(x, y),\left.\quad u_{t}(x, y, t)\right|_{t=0}=h(x, y),
\end{array}
$$

where

$$
g(x, y)= \begin{cases}\sin \left(2 \pi\left(x^{2}+y^{2}\right)\right), & 0 \leq x^{2}+y^{2} \leq 1 \\ 0, & x^{2}+y^{2}>1\end{cases}
$$



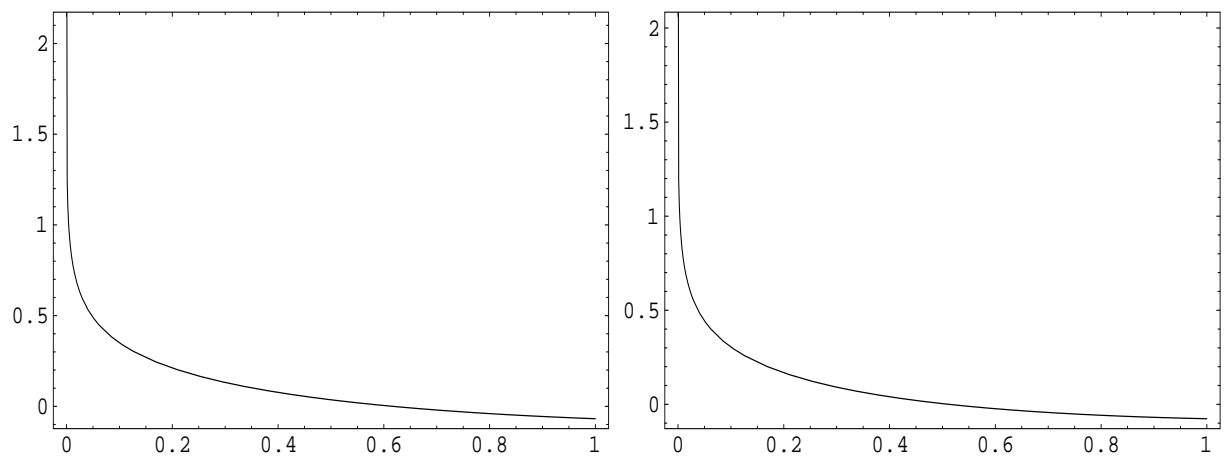

FIG. 5.1. $H Y_{0,2}^{\prime}(t)$ and $H Y_{1,2}^{\prime}(t)$
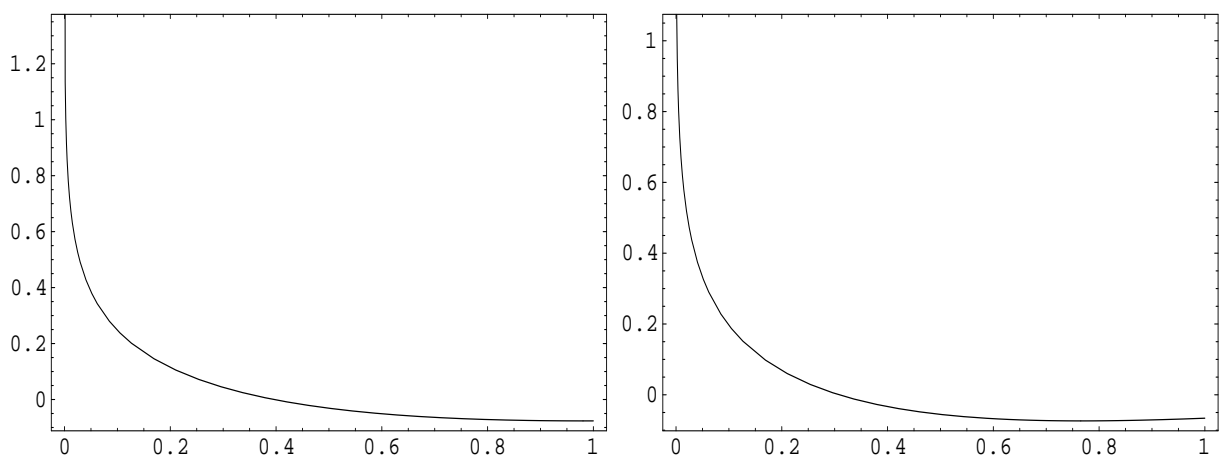

FiG. 5.2. $H Y_{2,2}^{\prime}(t)$ and $H Y_{3,2}^{\prime}(t)$

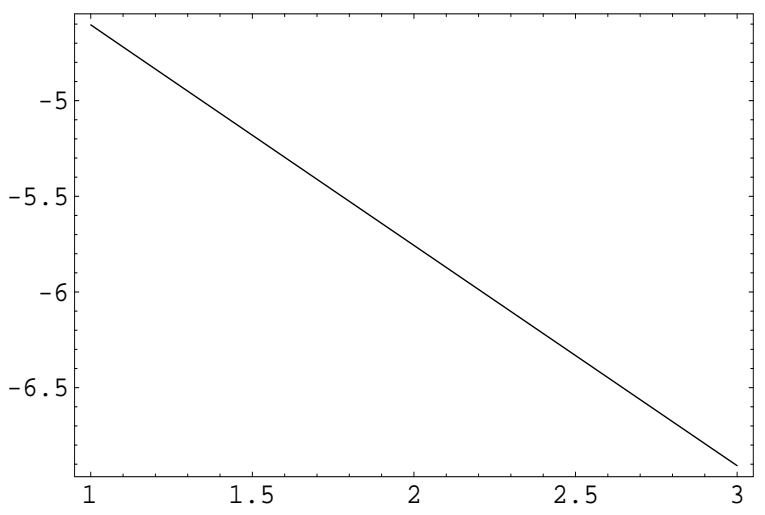

FIG. 5.3. The graph of $\left|C_{2}(k)\right|$ in logarithmic scales. Here $\Delta t=1 / 2000$.

$$
h(x, y)= \begin{cases}2 \pi \cos \left(2 \pi\left(x^{2}+y^{2}\right)\right), & 0 \leq x^{2}+y^{2} \leq 3 / 4 \\ 0, & x^{2}+y^{2}>3 / 4\end{cases}
$$

Thus, the initial condition only depends on $r$.

Since it is hard to find the exact solution of this problem, a highly-accurate 
numerical solution is needed to play this role, which is called "the accurate solution" in the following discussion. This solution is obtained by a standard second-order, centered difference discretization in space and time with mesh size $h=1 / 1024$, time step $\tau=h / 2$ and a N-order ABC-with $N=8$ in (2.40).

Next, we introduce an artificial boundary $\Gamma_{2} \times(0, T]$ and consider the problem in the bounded domain $\{(r, t) \mid 0 \leq r \leq 2,0<t \leq 3\}$. A linear finite element scheme with a lumping technique for the space directions is employed with a third order $\mathrm{ABC}$ in the numerical implementation at each time step. A second order centered difference with $\tau=h / 2$ is used to approximate the time derivatives, and the integral term for $H Y_{n, 2}(t)$ is approximated with a numerical quadrature scheme.

FIG. 6.1 shows the maximal error on $t=2$ and $t=3$ with different meshes.
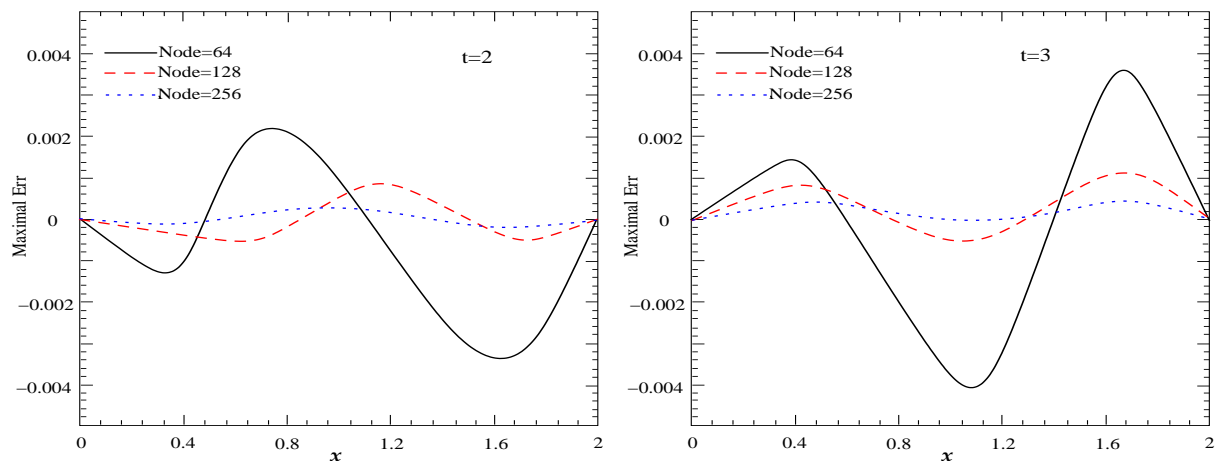

FIG. 6.1. Maximal error on time points, left is $t=2$, right is $t=3$

The relative error and convergence rates are shown in the TABLE 6.1. It can be observed that the errors decay with a nearly-optimal convergence rate of 4 when the mesh is refined by a factor 2 .

TABLE 6.1. The relative error and convergence rate of Example 1.

\begin{tabular}{ccccccc}
\hline Node & $t=1$ & \multicolumn{2}{c}{$t=2$} & \multicolumn{2}{c}{$t=3$} \\
\hline 32 & $3.3104 \mathrm{E}-2$ & $\cdots$ & $3.4321 \mathrm{E}-2$ & $\cdots$ & $3.4509 \mathrm{E}-2$ & $\cdots$ \\
64 & $8.1243 \mathrm{E}-3$ & 4.541 & $8.1428 \mathrm{E}-3$ & 4.231 & $8.2046 \mathrm{E}-3$ & 4.212 \\
128 & $2.0123 \mathrm{E}-3$ & 4.121 & $2.0314 \mathrm{E}-3$ & 4.102 & $2.0218 \mathrm{E}-3$ & 4.089 \\
256 & $5.0022 \mathrm{E}-4$ & 3.986 & $5.0321 \mathrm{E}-4$ & 3.954 & $5.0428 \mathrm{E}-4$ & 4.002 \\
512 & $1.2132 \mathrm{E}-4$ & 4.014 & $1.1254 \mathrm{E}-4$ & 3.988 & $1.2318 \mathrm{E}-4$ & 4.012 \\
\hline
\end{tabular}

Next, we consider the following Klein-Gordon equation

EXAMPLE 6.2.

$$
\begin{array}{r}
\frac{\partial^{2}}{\partial t^{2}} u(x, y, t)-\Delta u(x, y, t)+u(x, y, t)=0, \\
\left.u(x, y, t)\right|_{t=0}=g(x, y),\left.\quad u_{t}(x, y, t)\right|_{t=0}=h(x, y),
\end{array}
$$

with

$$
g(x, y)= \begin{cases}\left(1-x^{2}\right)\left(1-y^{2}\right), & 0 \leq|x|,|y| \leq 1 \\ 0, & \text { otherwise }\end{cases}
$$




$$
h(x, y)= \begin{cases}\sin 4 \pi((1-|x|)(1-|y|)), & 0 \leq|x|,|y| \leq 1, \\ 0, & \text { otherwise }\end{cases}
$$

The centered difference scheme is used to approximate the time derivatives. A Linear triangular finite element scheme with a lumping technique for the space directions at each time step is used with $\tau / h=0.5$, where $h$ denotes the mesh size. Figure 6.2 demonstrates the type of mesh used in our examples. The "accurate solution" is obtained with a mesh size of $h=1 / 1024$ and a twelfth order ABC.

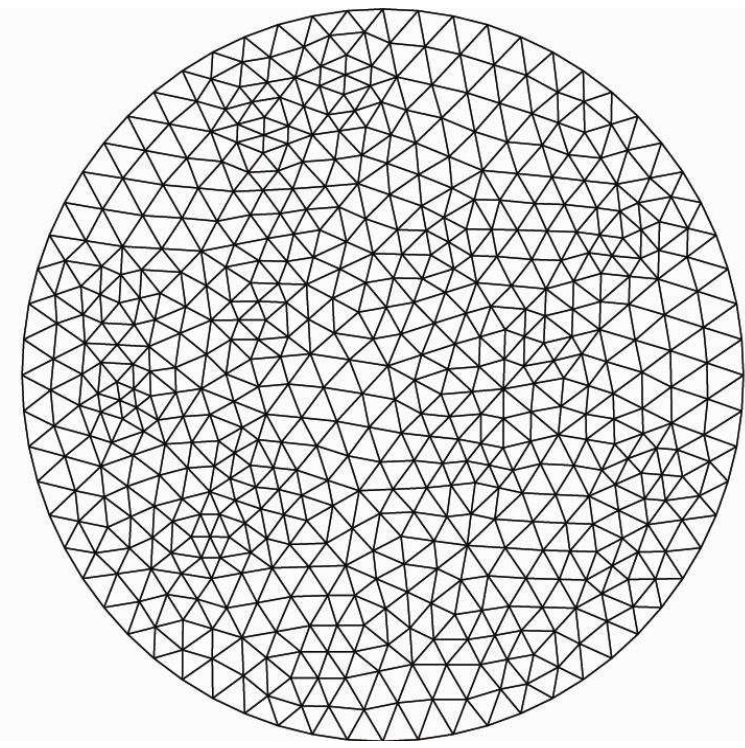

FIG. 6.2. Mesh plot with $h=1 / 16$

Figure 6.3 demonstrates the maximal error on $t=2$ and $t=3$ with different meshes along the line $y=0$.
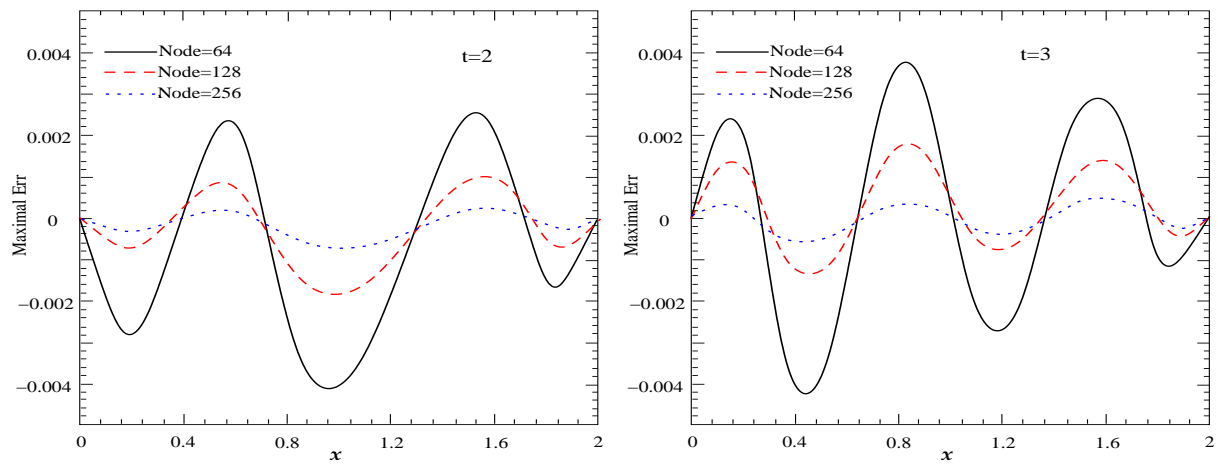

Fig. 6.3. Maximal error on $t=2,3$ along line $y=0$

Figure 6.4 shows the convergence rates of artificial boundary conditions with different accuracy, namely, with $N=1,2,4,6,7$. In FIG. 6.4, when $N$ is small, the 
accuracy of the numerical solutions can't be improved by refining the meshes because the artificial boundary conditions are not very accurate. When $N$ becomes large, the numerical solution approximates the "accurate solution" very well. When $N=7$, the convergence rate is almost two, which means the artificial boundary coundition with $N=7$ is a good approximation of the exact boundary condition.
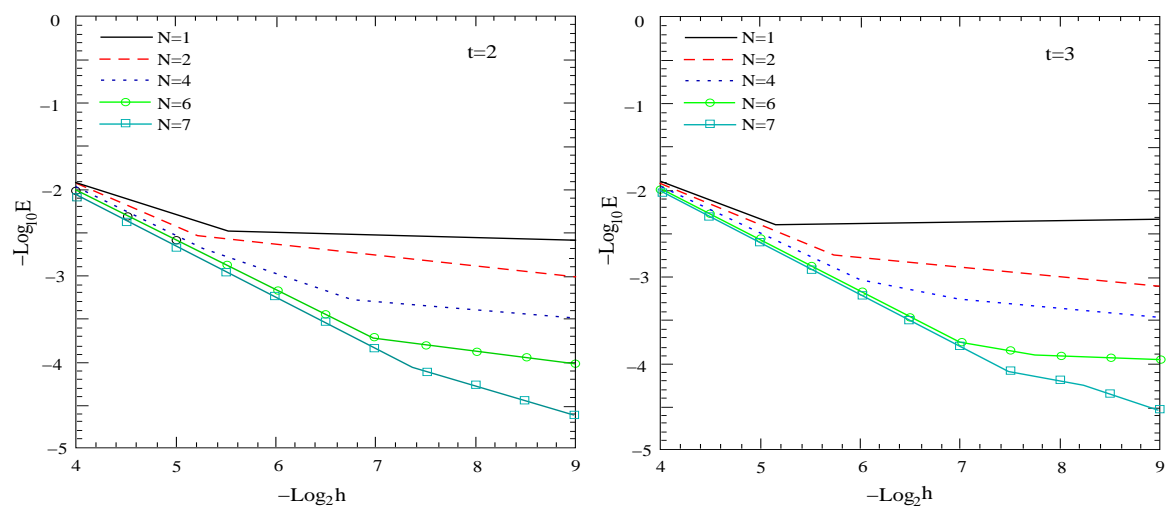

FIG. 6.4. The convergence rate of the artificial boundary conditions with different accuracy

In practical computation, the error of numerical solutions depends on the location of the artificial boundaries. To investigate the relation between the errors and the location of the artificial boundaries, we select different artificial boundaries, namely $R=2,3,4,5$ and $N=5$. The numerical error of at $(x, y)=(1.5,1.5)$ is shown in TABLE 6.2. We can see that the location of artificial boundaries has little effect on the accuracy of our artificial boundary conditions.

TABLE 6.2. The numerical results for different artificial boundaries

\begin{tabular}{ccccc}
\hline mesh size & $R=2$ & $R=3$ & $R=4$ & $R=5$ \\
\hline $1 / 16$ & $5.2602 \mathrm{E}-2$ & $4.8267 \mathrm{E}-2$ & $4.5481 \mathrm{E}-2$ & $4.3968 \mathrm{E}-2$ \\
$1 / 32$ & $1.3104 \mathrm{E}-2$ & $1.2102 \mathrm{E}-2$ & $1.1208 \mathrm{E}-2$ & $1.0682 \mathrm{E}-2$ \\
$1 / 64$ & $3.2368 \mathrm{E}-3$ & $3.0172 \mathrm{E}-3$ & $2.7149 \mathrm{E}-3$ & $2.5127 \mathrm{E}-3$ \\
$1 / 128$ & $7.9138 \mathrm{E}-4$ & $7.4873 \mathrm{E}-4$ & $6.7148 \mathrm{E}-4$ & $6.3163 \mathrm{E}-4$ \\
$1 / 256$ & $1.9143 \mathrm{E}-4$ & $1.8123 \mathrm{E}-4$ & $1.7032 \mathrm{E}-4$ & $1.5932 \mathrm{E}-4$ \\
\hline
\end{tabular}

There are two ways to improve the accuracy of the artificial boundary conditions; one way is to extend the computational domain. Another way is to increase $N$, the number of terms in the truncated artificial boundary conditions. For the same numerical accuracy, we compared the computational time of the two approaches. The mesh size was chosen to be $h=1 / 256$ and $t=3$. From the numerical results we can see that in order to save computational cost one can choose a smaller computational domain and bigger $N$, which is an advantage of our high accuracy artificial boundary conditions. 
TABLE 6.3. Computational time of different $R$ and $N$

\begin{tabular}{cccccccccc}
\hline accuracy & $R$ & $N$ & CPU time & $R$ & $N$ & CPU time & $R$ & $N$ & CPU time \\
\hline $1.0 \mathrm{e}-3$ & 2 & 4 & $2 \mathrm{~s}$ & 3 & 2 & $6 \mathrm{~s}$ & 4 & 1 & $19 \mathrm{~s}$ \\
$1.0 \mathrm{e}-4$ & 2 & 5 & $4 \mathrm{~s}$ & 3 & 3 & $13 \mathrm{~s}$ & 4 & 2 & $19 \mathrm{~s}$ \\
$1.0 \mathrm{e}-5$ & 2 & 7 & $9 \mathrm{~s}$ & 3 & 5 & $28 \mathrm{~s}$ & 4 & 4 & $70 \mathrm{~s}$ \\
\hline
\end{tabular}

\section{Conclusion}

The numerical simulation for the multidimensional Klein-Gordon equation is considered. By introducing an artificial boundary and giving either the exact or a family of approximate artificial boundary conditions, the original problem is reduced to an equivalent problem or a sequence of approximate problems, respectively, on the bounded computational domain. Furthermore, stability analysis of the approximate problem on a bounded computational domain is established. The performance of the numerical examples shows that the given method is feasible and effective.

\section{Appendix A. Proof of the formula used in ABCs.}

Lemma A.1. When $a>0, b>c, R>0, \nu \geq 0$, we have the following equalities

$$
\begin{aligned}
\int_{R}^{+\infty} J_{\nu}(a \xi) K_{\nu}(b \xi) \xi d \xi & =\frac{a^{2}+b^{2}}{R}\left\{b J_{\nu}(a R) K_{\nu+1}(b R)-a J_{\nu+1}(a R) K_{\nu}(b R)\right\}, \\
\int_{R}^{+\infty} Y_{\nu}(a \xi) K_{\nu}(b \xi) \xi d \xi & =\frac{R}{a^{2}+b^{2}}\left\{b Y_{\nu}(a R) K_{\nu+1}(b R)-a Y_{\nu+1}(a R) K_{\nu}(b R)\right\}, \\
-\frac{2}{\pi} \frac{K_{\nu}(r)}{K_{\nu}(R)} & =\int_{0}^{+\infty} \frac{\omega}{\omega^{2}+1} \frac{J_{\nu}(\omega r) Y_{\nu}(\omega R)-J_{\nu}(\omega R) Y_{\nu}(\omega r)}{J_{\nu}^{2}(\omega R)+Y_{\nu}^{2}(\omega R)} d \omega .
\end{aligned}
$$

Proof.

1. From the formula 6.521 in [9], we obtain

$$
\begin{aligned}
& \int_{R}^{+\infty} J_{\nu}(a \xi) K_{\nu}(b \xi) \xi d \xi \\
= & \int_{0}^{+\infty} J_{\nu}(a \xi) K_{\nu}(b \xi) \xi d \xi-\int_{0}^{R} J_{\nu}(a \xi) K_{\nu}(b \xi) \xi d \xi \\
= & \left(\frac{b}{a}\right)^{\nu} \frac{1}{a^{2}+b^{2}}- \\
& \frac{1}{a^{2}+b^{2}}\left\{\left(\frac{b}{a}\right)^{\nu}+a R J_{\nu+1}(a R) K_{\nu}(b R)-b R J_{\nu}(a R) K_{\nu+1}(b R)\right\} \\
= & \frac{R}{a^{2}+b^{2}}\left\{b J_{\nu}(a R) K_{\nu+1}(b R)-a J_{\nu+1}(a R) K_{\nu}(b R)\right\} .
\end{aligned}
$$

Thus, (A.1) is proved.

2. Let

$$
\begin{aligned}
& F_{1}(R)=\int_{R}^{+\infty} Y_{\nu}(a \xi) K_{\nu}(b \xi) \xi d \xi \\
& F_{2}(R)=\frac{R}{a^{2}+b^{2}}\left\{a Y_{\nu}(a R) K_{\nu+1}(b R)-a Y_{\nu+1}(a R) K_{\nu}(b R)\right\} .
\end{aligned}
$$


If we differentiate $F_{1}(R)$ and $F_{2}(R)$ with respect to $R$, we have

$$
\begin{aligned}
\frac{d F_{1}}{d R}= & -R Y_{\nu}(a R) K_{\nu}(b R), \\
\frac{d F_{2}}{d R}= & \frac{1}{a^{2}+b^{2}}\left\{b Y_{\nu}(a R) K_{\nu+1}(b R)-a Y_{\nu+1}(a R) K_{\nu}(b R)\right\} \\
& +\frac{R}{a^{2}+b^{2}}\left\{a b Y_{\nu}^{\prime}(a R) K_{\nu+1}(b R)+b^{2} Y_{\nu}(a R) K_{\nu+1}^{\prime}(b R)\right. \\
& \left.-a^{2} Y_{\nu+1}^{\prime}(a R) K_{\nu}(b R)-a b Y_{\nu+1}(a R) K_{\nu}^{\prime}(b R)\right\} \\
= & \frac{1}{R\left(a^{2}+b^{2}\right)}\left\{\left[R b Y_{\nu}(a R)+R^{2} a b Y_{\nu}^{\prime}(a R)\right] K_{\nu+1}(b R)\right. \\
& -\left[R a Y_{\nu+1}(a R)+R^{2} a^{2} Y_{\nu+1}^{\prime}(a R)\right] K_{\nu}(b R) \\
& \left.-R^{2} a b Y_{\nu+1}(a R) K_{\nu}^{\prime}(b R)+R^{2} b^{2} Y_{\nu}(a R) K_{\nu+1}^{\prime}(b R)\right\} .
\end{aligned}
$$

Using the formula [2]

$$
\begin{aligned}
& x Y_{\nu}^{\prime}(x)=x Y_{\nu-1}(x)-\nu Y_{\nu}(x), \\
& x Y_{\nu+1}^{\prime}(x)=x Y_{\nu}(x)-(\nu+1) Y_{\nu+1}(x), \\
& x K_{\nu}^{\prime}(x)=v K_{\nu}(x)-x K_{\nu+1}(x), \\
& x K_{\nu+1}^{\prime}(x)=-(\nu+1) K_{\nu+1}(x)-x K_{\nu}(x), \\
& x Y_{\nu-1}(x) x Y_{\nu+1}(x)-2 \nu Y_{\nu}(x)=0,
\end{aligned}
$$

we arrive at

$$
\frac{d F_{2}}{d R}=-R Y_{\nu}(a R) K_{\nu}(b R)=\frac{d F_{1}}{d R}
$$

and

$$
\lim _{R \rightarrow+\infty} F_{1}(R)=\lim _{R \rightarrow+\infty} F_{2}(R)=0 .
$$

Thus, (A.2) is proved.

3. From Weber-Orr's formula ([6], P.74), we obtain

$$
\begin{aligned}
f(r)= & \int_{0}^{+\infty} \frac{J_{\nu}(\omega r) Y_{\nu}(\omega R)-J_{\nu}(\omega R) Y_{\nu}(\omega r)}{\left[J_{\nu}(\omega R)\right]^{2}+\left[Y_{\nu}(\omega R)\right]^{2}} \omega d \omega \\
& \times \int_{0}^{+\infty}\left[J_{\nu}(\xi \omega) Y_{\nu}(R)-Y_{\nu}(\xi \omega) J_{\nu}(R)\right] \xi f(\xi) d \xi .
\end{aligned}
$$

(A.4) is correct for $\nu$ and $f(\xi)$ satisfying $\int_{0}^{+\infty} \xi^{\frac{1}{2}}|f(\xi)| d \xi<+\infty$. For a continuous point $\xi=r$ of $f(\xi)$, the right hand side of (A.4) is $f(r)$. If $f(\xi)$ is discontinuous at $\xi=r$, then the left side of (A.4) becomes $\frac{1}{2}(f(r-0)+f(r+0))$. Choose $f(\xi)$ as:

$$
f(\xi)=\left\{\begin{array}{l}
K_{\nu}(\xi), \xi \geq R, \\
0, \quad 0 \leq \xi<R .
\end{array}\right.
$$

Obviously, $f(\xi)$ satisfies the conditions for $\int_{0}^{+\infty} \xi^{1 / 2}|f(\xi)| d \xi<+\infty$. 
Combining (A.1) and (A.2), we obtain

$$
\begin{aligned}
& \int_{0}^{+\infty}\left[J_{\nu}(\xi \omega) Y_{\nu}(\omega R)-Y_{\nu}(\xi \omega) J_{\nu}(\omega R)\right] \xi f(\xi) d \xi \\
= & Y_{\nu}(\omega R) \int_{R}^{+\infty} J_{\nu}(\xi \omega) K_{\nu}(\xi) \xi d \xi-J_{\nu}(\omega R) \int_{R}^{+\infty} Y_{\nu}(\xi \omega) K_{\nu}(\xi) \xi d \xi \\
= & Y_{\nu}(\omega R) \frac{R}{\omega^{2}+1}\left[J_{\nu}(\omega R) K_{\nu+1}(R)-\omega J_{\nu+1}(\omega R) K_{\nu}(R)\right] \\
& -J_{\nu}(\omega R) \frac{R}{\omega^{2}+1}\left[Y_{\nu}(\omega R) K_{\nu+1}(R)-\omega Y_{\nu+1}(\omega R) K_{\nu}(R)\right] \\
= & \frac{R \omega}{\omega^{2}+1}\left[-Y_{\nu}(R \omega) J_{\nu+1}(\omega R)+J_{\nu}(R \omega R) Y_{\nu+1}(\omega R)\right] K_{\nu}(R) \\
= & \frac{R \omega}{\omega^{2}+1}\left[Y_{\nu}(\omega R) J_{\nu}^{\prime}(\omega R)-J_{\nu}(\omega R) Y_{\nu}^{\prime}(\omega R)\right] K_{\nu}(R) \\
= & -\frac{R \omega}{\omega^{2}+1} \frac{2}{\pi} \frac{1}{R \omega}=-\frac{2}{\pi} \frac{1}{\omega^{2}+1} .
\end{aligned}
$$

The second to last formula is a consequence of the Wronskian determination of $J_{\nu}(x), Y_{\nu}(x)[2]$.

By substituting (A.6) into (A.5), the formula (A.3) is proved.

\section{REFERENCES}

[1] B. Alpert, L. Greengard and T. Hagstrom, Rapid evaluation of nonreflecting boundary kernels for time-domain wave propagation, SIAM J. Numer. Anal., 37, 1138-1164, 2000.

[2] L.C. Andrews, Special Functions of Mathematics for Engineers, McGraw-Hill, New York, NY (United States), 1992.

[3] A. Bayliss and E. Turkel, Radiation boundary conditions for wave like equations, Comm. Pure. Appl. Math., 23, 707-725, 1980.

[4] J.P. Berenger, A perfectly matching layer for the absorbing of electromagnetic waves, J. Comput. Phys., 114, 185-200, 1994.

[5] B. Engquist and A. Majda, Absorbing boundary conditions for the numerical simulation of waves, Math. Comput., 31, 629-651, 1977.

[6] A. Erdélyi, Tables of Integral Transforms, Vol II, McGraw-Hill, 1954.

[7] D. Givoli, Non-reflecting boundary conditions: a review, J. Comput. Phys., 94, 1-29, 1991.

[8] D. Givoli, High-order local non-reflecting boundary conditions: a review, Wave Motion, 39, 319-326, 2004.

[9] I.S. Gradshteyn and I.M. Ryzhik, Table of Integrals, Series and Products, sixth edition, Academic Press.

[10] W. Greiner, Relativistic Quantum Mechanics-wave Equations, third edtion, Springer, 2000.

[11] M.J. Grote and J.B. Keller, Exact nonreflecting boundary conditions for the time dependent wave equation, SIAM J. Appl. Math., 55, 280-297, 1995.

[12] T. Hagstrom, Radiation boundary conditions for the numerical simulation of waves, Acta. Numerica, 8, 47-106, 1999.

[13] H. Han and Z. Huang, Exact artificial boundary conditions for the Schrödinger equations in $\mathbb{R}^{2}$, Commun. Math. Sci., 2(1), 79-94, 2004.

[14] H. Han and C.X. Zheng, Exact nonreflecting boundary conditions for exterior problems of the hyperbolic equation, Chinese J. Comput. Phys., 22(2), 95-107, 2005.

[15] H. Han and C.X. Zheng, Exact nonreflecting boundary conditions for acoustic problem in three dimensions, J. Comput. Math., 21, 15-24, 2003.

[16] H. Han, D.S. Yin and Z. Huang, Numerical solutions of Schrödinger equations in $\mathbb{R}^{3}$, Numerical Methods for Partial Differential Equations, 23(3), 511-533, 2007.

[17] R.L. Higdon, Absorbing boundary conditions for difference approximation to the multidimensional wave equation, Math. Comput., 47, 437-459, 1986. 
[18] R. Kosloff and D. Kosloff, Absorbing boundaries for wave propagation problems, J. Comput. Phys., 63, 363-376, 1986.

[19] C. Lubich and A. Schädle, Fast convolution for nonreflecting boundary conditions, SIAM J. Sci. Comput., 24, 161-182, 2002.

[20] I.L. Sofronov, Conditions for complete transparency on the sphere for the three-dimensional wave equation, Russian. Acad. Sci. Dokl, 46, 397-401, 1993.

[21] Z.H. Teng, Exact boundary condition for time-dependent wave equation based on boundary integral, J. Comput. Phys., 190, 398-418, 2003.

[22] L. Ting and M.J. Miksis, Exact boundary conditions for scattering problems, J. Acoust. Soc. Amer., 80, 1825-1827, 1986. 